\title{
Schizophrenia-associated HapICE haplotype is associated with increased NRG1 type III expression and high nucleotide diversity
}

\author{
CS Weickert ${ }^{1,2,3}$, Y Tiwari ${ }^{1,3,4}$, PR Schofield ${ }^{1,3,4}$, BJ Mowry ${ }^{5,6}$ and JM Fullerton ${ }^{1,3,4}$
}

Excitement and controversy have followed neuregulin (NRG1) since its discovery as a putative schizophrenia susceptibility gene; however, the mechanism of action of the associated risk haplotype (HapICE) has not been identified, and specific genetic variations, which may increase risk to schizophrenia have remained elusive. Using a postmortem brain cohort from 37 schizophrenia cases and 37 controls, we resequenced upstream of the type I-IV promoters, and the HapICE repeat regions in intron 1. Relative abundance of seven NRG1 mRNA transcripts in the prefrontal cortex were determined and compared across diagnostic and genotypic groups. We identified 26 novel DNA variants and showed an increased novel variant load in cases compared with controls $\left(\chi^{2}=7.815 ; P=0.05\right)$. The average nucleotide diversity $\left(\theta=10.0 \times 10^{-4}\right)$ was approximately twofold higher than that previously reported for BDNF, indicating that NRG1 may be particularly prone to genetic change. A greater nucleotide diversity was observed in the HapICE linkage disequilibrium block in schizophrenia cases $\left(\theta_{\text {(case) }}=13.2 \times 10^{-4}\right.$; $\left.\theta_{\text {(control) }}=10.0 \times 10^{-4}\right)$. The specific HapICE risk haplotype was associated with increased type III mRNA $(F=3.76, P=0.028)$, which in turn, was correlated with an earlier age of onset $(r=-0.343, P=0.038)$. We found a novel intronic five-SNP haplotype $\sim 730 \mathrm{~kb}$ upstream of the type I promoter and determined that this region functions as transcriptional enhancer that is suppressed by SRY. We propose that the HapICE risk haplotype increases expression of the most brain-abundant form of NRG1, which in turn, elicits an earlier clinical presentation, thus providing a novel mechanism through which this genetic association may increase risk of schizophrenia.

Translational Psychiatry (2012) 2, e104; doi:10.1038/tp.2012.25; published online 17 April 2012

\section{Introduction}

Neuregulin-1 (NRG1), a pluripotent regulator of neurodevelopment, ${ }^{1-5}$ has shown genetic association with the diagnosis of schizophrenia in many but not all populations from around the world, ${ }^{6,7}$ implicating SNP polymorphisms in both the $5^{\prime}$ and $3^{\prime}$ regions. ${ }^{8,9}$ Meta-analysis of 22 studies has demonstrated replication at the level of the haplotype but not at the single SNP level, ${ }^{7,10}$ and have highlighted significant between study heterogeneity, suggesting that specific risk alleles are likely to vary across populations and individuals.

Association of NRG1 genotypes with a variety of phenotypes, including mRNA expression levels, brain volume, brain activation and eye movements have been identified. ${ }^{11-17}$ Furthermore, the high affinity tyrosine kinase receptor, ErbB4, which partners with NRG1 to control neurodevelopment, ${ }^{2,4,5}$ is also associated with schizophrenia at both genetic and transcriptional levels. ${ }^{18-22}$ Thus, at least two key components of a neurodevelopmental signaling pathway have been strongly implicated in the etiology of schizophrenia.
The mechanism by which variation in NRG1 and ErbB4 increases risk for schizophrenia is unknown. Available evidence suggests that splice variant specific NRG1/ErbB4 overexpression ${ }^{11,13,18,21,23}$ and hypersignaling of NRG1 ${ }^{24}$ may be involved, although some reports of reduced or unchanged expression of NRG1 mRNA or protein (alpha form) in the brain and serum also exist. ${ }^{23,25,26}$ Specific mRNA transcripts of NRG1 $1^{11,13,15,23}$ and ErbB4 $4^{18,19,21}$ are increased in the frontal cortex and hippocampus of patients with schizophrenia and variable NRG1 transcript expression has been linked to promoter and intronic polymorphisms associated with schizophrenia; ${ }^{11,13,15}$ however, the increase in cortical NRG1 type I mRNA ${ }^{11,13}$ has not been replicated in an independent Caucasian cohort ${ }^{23}$ and the relationship of mRNA changes to the haplotype associated with increased risk of a clinical diagnosis of schizophrenia ${ }^{6,8,27}$ has not been defined. In addition, previous postmortem genetic studies of $N R G 1^{11,13,15}$ have been conducted in cohorts of mixed ethnicity (largely African American), with no ethnic matching to account for differences in genetic diversity across the populations.

\footnotetext{
${ }^{1}$ Schizophrenia Research Institute, Sydney, NSW, Australia; ${ }^{2}$ University of New South Wales, School of Psychiatry, Sydney, NSW, Australia; ${ }^{3} \mathrm{Psychiatric}$ Genetics, Neuroscience Research Australia, Sydney, NSW, Australia; ${ }^{4}$ University of New South Wales, School of Medical Sciences, Sydney, NSW, Australia; ${ }^{5}$ Queensland Centre for Mental Health Research, Brisbane, QLD, Australia; ${ }^{6}$ Queensland Brain Institute, University of Queensland, Brisbane, QLD, Australia Correspondence: Dr JM Fullerton, Psychiatric Genetics, Neuroscience Research Australia, Barker Street, Randwick, Sydney, NSW 2031, Australia. E-mail: j.fullerton@neura.edu.au

Keywords: dorsolateral prefrontal cortex; HapICE; NRG1 isoform expression; postmortem brain; schizophrenia

Received 28 October 2011; revised 9 February 2012; accepted 3 March 2012
} 
In this study, we first characterized the nucleotide diversity in promoter regions of NRG1 in postmortem brain tissue of 37 controls and 37 patients with schizophrenia, most of Australian Caucasian ethnicity. We then tested whether DNA variants were in linkage disequilibrium (LD) with the schizophrenia-associated risk haplotype (HaplCE) ${ }^{8,27}$ and whether they were functional. Lastly, we determined if differences in seven NRG1 mRNA transcripts were evident in the frontal cortex of schizophrenia in this independent postmortem cohort, and whether NRG1 gene variants are associated with variation in mRNA expression.

\section{Materials and methods}

Human postmortem cohort and tissue extraction. Standard protocols ${ }^{28}$ were used to extract genomic DNA and total RNA from the dorsolateral prefrontal cortex (DLPFC) of 74 fresh frozen postmortem brains available from the Tissue Resource Center, Sydney, Australia. The demographic characteristics, tissue processing and quality screening for this cohort is described elsewhere, ${ }^{29}$ and ascertainment and exclusions are described in brief in Supplementary Material. Case $(n=37)$ and control samples $(n=37)$ were matched for age, brain $\mathrm{pH}$, RNA integrity, hemisphere, gender and postmortem interval (Supplementary Table 1).

Detection of nucleotide variation in NRG1 putative regulatory regions. To characterize the nucleotide diversity in the alternative promoter regions of NRG1 in DNA from the DLPFC of the human postmortem brain cohort, we resequenced $\sim 3 \mathrm{~kb}$ upstream of each transcription start site of the type IV/II, type I and type III isoforms via Bigdye terminator sequencing (Applied Biosystems, Melbourne, Victoria, Australia) (Figure 1). In addition, we sequenced the polymorphisms constituting the 5-SNP and 7-marker HapICE risk haplotypes ${ }^{8}$ (SNP8NRG221132, SNP8NRG221533, SNP8NRG241930, SNP8NRG243177 and SNP8NRG433E1006, plus microsatellite repeats 478B14-848 and 420M9-1395). Further details of SNP calling and bioinformatic analysis are provided in supplementary material.

Australian control cohort. To estimate the prevalence of a novel 5-SNP haplotype in the Australian population, 128 control individuals ascertained through the National prevalence study of psychosis ${ }^{30}$ were screened. Further details are given in supplementary material.

Quantification of NRG1 isoform expression. Relative transcript levels were measured for each cDNA sample in triplicate by real-time quantitative RT-PCR and normalized to the geometric mean of four endogenous control genes, which did not differ between diagnostic groups. Details on quantitative PCR probes, methods and quality control are given in supplementary methods and Supplementary Table 2.

Functional characterization of genetic polymorphisms. Luciferase assays were performed in HEK293 cells using the Dual-Luciferase Reporter Assay System (Promega, Sydney, New South Wales, Australia) as per manufacturers' instructions. DNA amplified from individuals with either a wild-type or novel 5-SNP haplotype was cloned upstream of the firefly luciferase reporter gene in pGL3B. Transfection efficiency was monitored and normalized by cotransfection with renilla luciferase. As gene variants in the novel haplotype were bioinformatically predicted to alter SRY transcription factor-binding efficiency, full-length human SRY cDNA (kindly donated by Prof. Andrew Sinclair) cloned into pcDNA4.1 was used for cotransfection.

Electrophoretic mobility shift assays were conducted to determine whether alternative alleles affected transcription

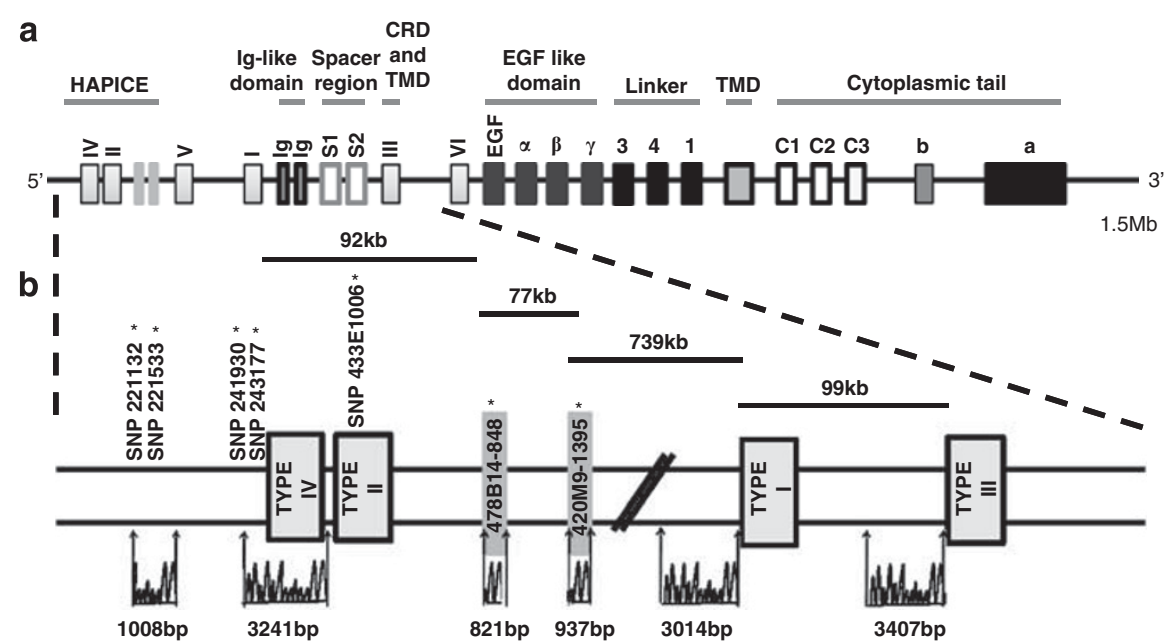

Figure 1 Genomic structure and location of resequenced regions of human NRG1. (a) Human NRG1 is transcriptionally complex, with over 30 known mRNA splicing isoforms that are mainly driven off four alternative promoters (I-IV) and two minor promoters (V-VI). Alternative isoforms include different functional protein domains, with type III isoform containing two transmembrane domains (TMD) and a cysteine-rich domain (CRD). Proteolytic cleavage of type I and III isoforms produces a C-terminal fragment (CTF) and N-terminal fragment (NTF) containing a bioactive EGF domain. Downstream processing by $\gamma$-secretase processes the CTF to release the intracellular domain (ICD) that contains the CRD. The position of the schizophrenia-associated HapICE haplotype is shown in the upstream promoter region. (b) The relative locations and sizes of resequenced regions are shown and the positions of SNP and microsatellite markers that constitute the HapICE haplotype are indicated. 
factor binding in vitro. Reaction conditions and protocols were as described previously. ${ }^{31}$

Statistical analysis. Statistical analyses were conducted using Statistica software (StatSoft, 2000; Tulsa, OK, USA). Both Pearson-product moment and Spearman rho correlations were calculated for relationships between brain cohort characteristics (demographic and clinical) and normalized NRG1 mRNA expression. Effects of dichotomous variables (gender and hemisphere) were examined by $t$-tests. Overall tests of normalized mRNA expression differences and genotype effects were analyzed by one-way and two-way ANCOVAs, taking into account any significantly correlated demographic variables.

\section{Results}

SNP discovery from deep targeted resequencing. In total, over $919 \mathrm{~kb}$ of good quality DNA sequence was available for SNP discovery (12.5 kb per sample), generated from the DNA extracted from the DLPFC of 74 human postmortem brains. From this sequence, 65 DNA variants were identified (Supplementary Tables 3 and 6), including 26 variants, which had not previously been characterized (dbSNP130; Supplementary Figure 1 and Supplementary Table 5). The novel DNA variants were relatively rare (minor allele frequency $=0.044 \pm 0.038$ ), and twice as likely to be found exclusively in schizophrenia cases as compared with controls (11 vs 5 novel SNPs; Supplementary Table 3). The novel DNA variants were more likely to be functional than previously known SNPs-85\% were predicted in silico to alter putative transcription factor-binding sites, compared with $65 \%$ of the previously characterized SNPs observed in our sample. Furthermore, a significantly higher number of schizophrenia patients had at least one novel variant compared with controls ( $n=24$ vs $\left.14 ; \chi^{2}=10.8, P=0.001\right)$, and the novel variant load in each individual was greater in schizophrenia cases compared with controls (Fishers exact test: $\chi^{2}=7.815 ; P=0.05$; Supplementary Figure 2).

Nucleotide diversity in NRG1 and relationship to schizophrenia diagnosis and HapICE. Overall, the promoter and intronic regions of NRG1 had an average nucleotide diversity $(\theta)$ of $10.0 \times 10^{-4}$ (Supplementary Table $3)$, which is higher than the average found in promoter and intronic regions of BDNF $\left(\theta=6.0 \pm 2.0 \times 10^{-4}\right)^{32}$ and 75 other comparator genes $\left(5.4 \pm 1.5 \times 10^{-4}\right) .{ }^{33}$ We found the lowest relative nucleotide diversity in the proximal promoter IV/II region $\left(\theta=11.1 \times 10^{-4}\right)$ and the highest nucleotide diversity around the HapICE microsatellite repeats in intron 1 $\left(\theta=26.8 \times 10^{-4}\right.$ and $30.1 \times 10^{-4}$, respectively, for 478B14848 and 420M9-1395). A greater nucleotide diversity was observed in cases compared with controls, which was largely driven by differences in the HapICE LD block (Figure 2); however, the majority of these novel variants occurred in individuals not carrying the specific HapICE risk haplotype. A cluster of five novel SNPs was identified in 4 of the 37 schizophrenia cases (frequency $=0.054$ ), but none of the 37 controls. These novel SNPs run as a haplotype in a

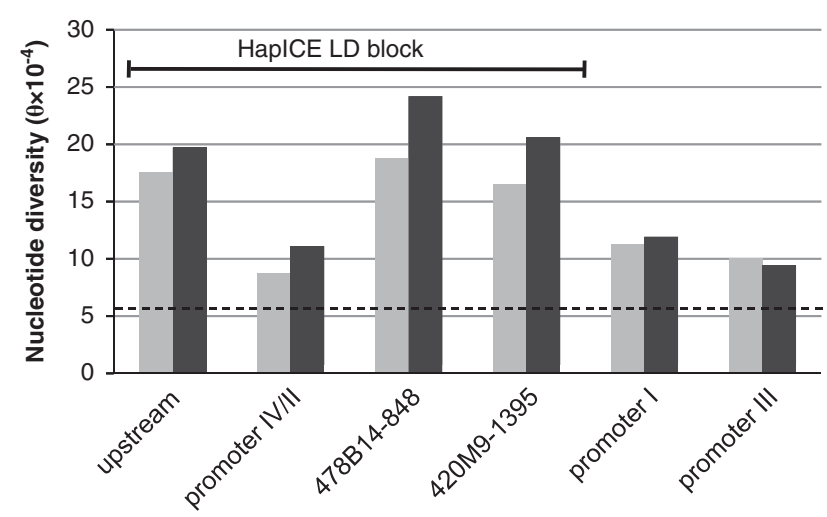

Figure 2 Nucleotide diversity in NRG1 regions comparing 37 controls (light gray) with 37 schizophrenia patients (dark gray). The dashed line indicates the average nucleotide diversity from non-coding regions of 75 other human genes ${ }^{33}$ at $\theta=5.4 \times 10^{-4}$. The regions that fall within the HapICE LD block are indicated.

single LD block (NCBI build 36: 31643-31875kb) surrounding the HapICE microsatellite repeats in intron 1. As this novel 5-SNP haplotype was relatively common in our cases, we further explored its frequency in peripheral blood samples from an independent psychiatrically assessed Australian control population and found it to be rare (1/256 chromosomes; frequency $=0.0039)$, and not brain specific.

Frequency comparisons of HapICE risk haplotype. To determine the frequency of the HapICE risk haplotype in the Australian postmortem cohort, genotypes were phased using PLINK and haplotypes with the best posterior probability of being correct were selected (mean $P$-value $=0.91 \pm 0.15$ ). Frequencies of the 5-SNP and 7-marker risk haplotypes were comparable between our Australian cohort (5-SNP: $32.4 \%$; 7-marker: $12.2 \%$ ) and those reported in the Scottish and Icelandic populations (5-SNP: 31.4 and 28.6\%; 7-marker: 8.0 and $11.0 \%$, respectively). ${ }^{27}$ No significant frequency distortion of either haplotype was observed in cases compared with controls $(P>0.844)$; however, this is likely because of the limited statistical power of our postmortem cohort to detect genetic associations.

Demographic factors and NRG1 mRNA expression. The type IV and EGF $\alpha$ isoforms were at the limit of detection because of their very low expression in the DLPFC, and hence their quantification was deemed unreliable. However, because of the interest in the type IV isoform in the literature, we included those results in Supplementary Material, but caution must be used in any interpretation.

Significant correlations with RNA integrity values were observed for all isoforms excluding type $\mathrm{I}_{(\mathrm{Ig} 2)}$ at the $P<0.05$ level, and significant correlations with brain $\mathrm{pH}$ were observed for pan-NRG1, EGF $\beta$, type II and type III mRNA (Supplementary Table 4). In addition, significant correlations with age and postmortem interval were observed for pan-NRG1 mRNA, and postmortem interval only for type $I_{(\lg 2)}$. All statistical comparisons included significant demographic variables as covariates in an ANCOVA test. No sex effects were observed for any robustly expressed NRG1 isoform in the DLPFC. 
NRG1 genotype and NRG1 isoform expression. To determine if the HapICE haplotype affected the expression of NRG1 mRNA, we tested the seven NRG1 transcripts against copies of the risk haplotype. We found a significant main effect of the 5-SNP haplotype on type III isoform expression (ANCOVA $\mathrm{F}(2,69)=3.763, P=0.028$, Figure $3 \mathrm{a}$ ), suggesting an allele dose response of type III expression with genetic risk. This effect was found in both cases and controls, and no main effect of diagnosis or haplotype $\times$ diagnosis was observed $(P>0.770)$. Hence, the LD block marked by the HapICE risk haplotype may contain a longrange enhancer of type III expression. There was no significant effect of HapICE risk haplotype on expression of other NRG1 isoforms (data not shown).

Next, we determined if any individual SNPs impacted NRG1 transcript levels. Because of constraints of sample size and statistical power, only SNPs with minor allele frequency of $\geqslant 25 \%$ were assessed. On this basis 11 of the 65 detected SNPs, including rs6994992 and rs7014762 that were previously reported to affect isoform expression, ${ }^{13,15}$ were tested for main effects of genotype plus genotype $\times$ disease interactions.

Three individual HapICE SNPs showed significant or suggestive effects on type III isoform expression: SNP8NRG221533 $(\mathrm{F}(2,68)=3.148, P=0.05)$; SNP8NRG241930 $(\mathrm{F}(2,66)=3.647, \quad P=0.032) ; \quad$ and $\quad$ SNP8NRG243177 $(\mathrm{F}(2,69)=2.919, P=0.061)$, with no genotype $\times$ diagnosis interactions observed $(P$-values all $>0.58)$. These SNPs lie in a single LD block over $1 \mathrm{Mb}$ upstream of the type III promoter and are consistent with the haplotype findings.

Although we did not observe a significant effect of rs7014762 genotype on type III expression $(F(2,66)=1.587$, $P=0.212$ ), the effect was in the same direction as that previously reported ${ }^{15}$ - where the minor allele predicts decreased type III expression (mean values for each genotype group $(n=6,30,38)$ were $3.01 \pm 0.43,3.77 \pm 0.19,3.95 \pm$ 0.116 , respectively). Unlike the previous study, ${ }^{15}$ we did not detect a genotype $\times$ diagnosis interaction for rs7014762 and type III mRNA $(\mathrm{F}(6,132)=0.394, P=0.676)$. Further exploration of rs7014762 in the context of the HapICE haplotype showed that the major $(\mathrm{C})$ allele lies on the specific risk haplotype (Supplementary Figure 3).
A second cluster of SNPs in intron 1 affected type II isoform expression. Significant genotype $\times$ diagnosis effects were observed for four individual SNPs: rs13263989, rs13253310, rs3802160 and rs36213229 ( $P$-values all 0.01-0.05). Two of those individual SNPs lie in a $77 \mathrm{~kb}$ LD block (NCBI build 36 : $31708-31785 \mathrm{~kb}$ ), which lies over $90 \mathrm{~kb}$ downstream of the type II promoter, consisting of SNPs rs13263989, rs13253310, rs11785744, the 478B14-848 microsatellite risk allele (14 repeats) and rs34150028. Individuals carrying the haplotype $\operatorname{CCC}(14) G$ showed a significant increase in type II expression $(F(1,65)=4.051, P=0.048$; Figure $3 b)$. It should be noted that none of these findings would survive strict Bonferroni correction (corrected $P=0.0023$ for 21 tests, for effects of 11 SNPs in three independent LD blocks on seven transcripts).

Diagnosis and brain hemisphere effect on NRG1 splice variant gene expression. We found no main effect of diagnosis on any NRG1 isoform measured (all $F<2.0$, all $P>0.05)$. Most of the NRG1 isoforms did not show hemispheric differences in expression, with the exception of pan-NRG1 and EGF $\beta$ mRNAs. For pan-NRG1, we observed higher expression in the left as compared with the right prefrontal cortex for both cases and controls $(P<0.03)$. For EGF $\beta$, we detected a significant diagnosis by hemisphere interaction $(\mathrm{F}(1,66)=4.00, P=0.049)$. Hemispheric asymmetry in EGF $\beta$ expression was observed in normal controls, where the left hemisphere has $23 \%$ more EGF $\beta$ mRNA as compared with the right $(P=0.019)$. Individuals with schizophrenia had significantly less EGF $\beta$ mRNA in the left hemisphere as compared with controls $(P=0.040)$, thus there appears to be a loss of the normal hemispheric asymmetry in EGF $\beta$ expression in the disease state (Supplementary Figure 4).

Clinical factors and NRG1 mRNA expression. Clinical factors including age of onset, duration of illness, smoking habit, anti-depressant history, daily chlorpromazine (antipsychotic) use, last chlorpromazine use and life-time chlorpromazine levels were examined for their effect on NRG1 mRNA. Age of onset was found to have significant correlation specifically with NRG1 type III isoform mRNA
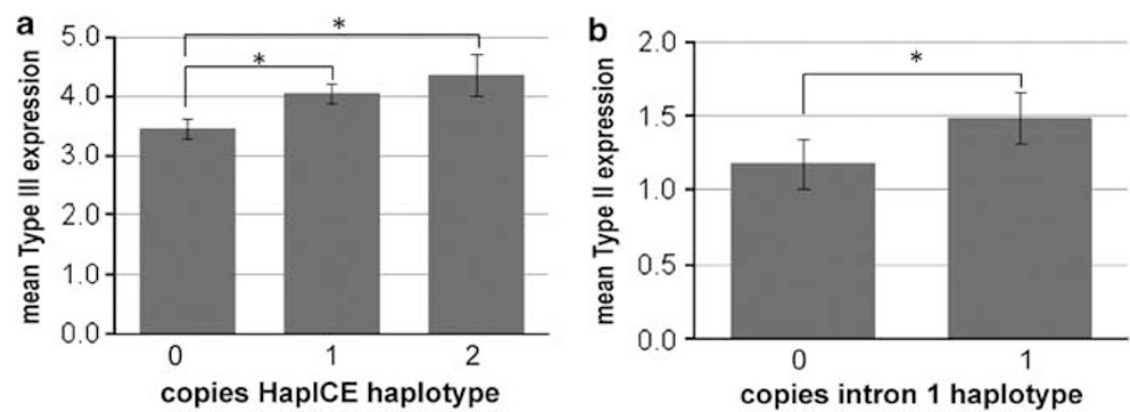

Figure 3 The effect of the HapICE and intron 1 haplotypes on NRG1 isoform expression. (a) The HapICE 5 SNP risk haplotype GCGTG has a significant effect on type III isoform expression (ANCOVA F $(2,69)=3.763, P=0.028$; post-hoc LSD: $P=0.013478$ or 0.019014 (for 1 or 2 copies compared with 0 copies)) with mean expression values ( \pm s.e.) for individuals $(n)$ with 0 copies $(n=34), 1$ copy $(n=32)$ or 2 copies $(n=8)$ were $3.446 \pm 0.168,4.048 \pm 0.173,4.349 \pm 0.357$, respectively. (b) The intron 1 haplotype CCC(14)G has a significant effect on type II expression (ANCOVA $F(1,65)=4.051, P=0.048$ ), with mean expression values $( \pm \mathrm{s}$.d.) for individuals $(n)$ with 0 copies $(n=46)$ or 1 copy $(n=26)$ were $1.18 \pm 0.56$ and $1.49 \pm 0.56$, respectively. No homozygotes for the $\operatorname{CCC}(14) G$ haplotype were observed. 

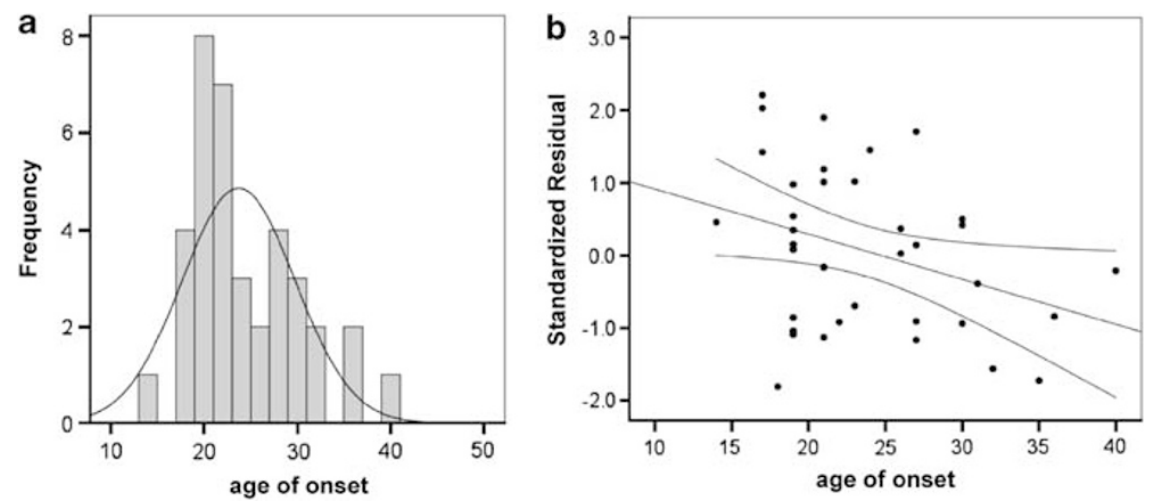

Figure 4 Age of onset at first diagnosis in postmortem brain cohort. (a) Frequency plot of age distribution in the sample, with age of onset on the $x$ axis and frequency on the $y$ axis. (b) Correlation of age of onset with NRG1 type III expression. Type III expression is presented on the $y$ axis as the standardized residual from the regression equation after accounting for the significant demographic effects of $\mathrm{pH}$ and postmortem interval (PMI) on expression. The linear regression line showing a significant correlation $(r=-0.343, P=0.038)$ is shown (black line) along with the mean $95 \%$ confidence intervals (black curved lines).

levels $(r=-0.343, \quad P=0.038 ; \quad$ Figure 4$)$, suggesting increased expression of NRG1 type III isoform relates to an earlier age of onset of schizophrenia in our Australian cohort. Duration of illness was positively correlated with type II mRNA ( $r=0.341, P=0.041)$, suggesting an upregulation of type II mRNA may occur with disease progression. This effect disappeared when corrected for age at death $(r=0.035$, $P=0.843$ ) although age at death was only significantly correlated with type II expression in cases $(r=0.342$, $P=0.048)$ and not controls $(r=-0.738, P=0.669)$, indicating that the age effect on type II expression may be disease specific. No significant correlations were found for any other isoform and age of onset, duration of illness (partial correlations with age at death), or with any measure of antipsychotic exposure.

Functional characterization of the unique 5-SNP haplotype in intron 1. Three of the novel SNPS that formed the novel 5-SNP haplotype were predicted in silico to reduce SRY transcription factor binding. To determine whether the two microsatellite regions that contained these novel SNPs could affect gene transcription, $\sim 1 \mathrm{~kb}$ of sequence surrounding each repeat was cloned into a pGL3B expression vector, from both a wild-type control individual and a schizophrenic individual, who carried the novel 5-SNP haplotype. In HEK293 cells (derived from an XX female), the wild-type 478B14-848 construct did not induce luciferase expression over baseline, however, a fivefold induction of expression was observed with the 420M9-1395 construct irrespective of genotype, indicating this region acts as a transcriptional enhancer (Figure 5). By cotransfecting human SRY (encoded on the male $Y$ chromosome), the efficiency of the 420M9-1395 intronic region to drive transcription was reduced by around 15\% (Figure 5), and the combination of both SRY and the novel haplotype further reduced expression over wild type at a trend level of significance $(t=1.7675, \mathrm{df}=9, P=0.055)$. Electrophoretic mobility shift assays experiments demonstrated that the minor allele of one novel variant in 420M9-1395 (1395_1) showed reduced nuclear protein binding in comparison with

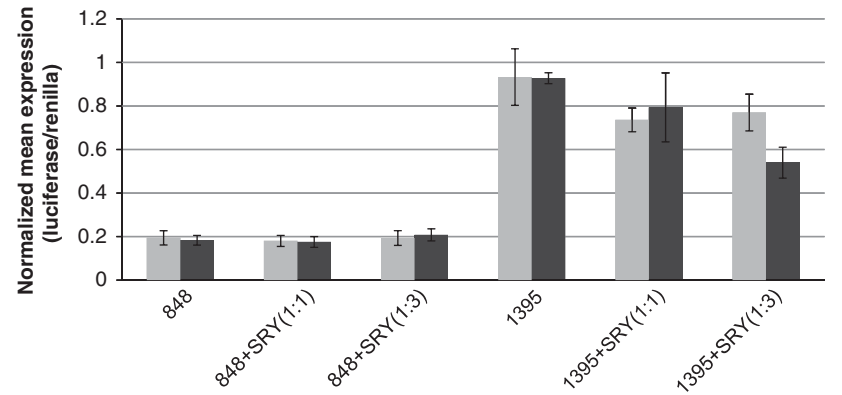

reporter construct

Figure 5 NRG1 intron 1 contains a transcriptional enhancer, which is repressed by the transcription factor SRY. HEK293 cells were transfected with $300 \mathrm{ng}$ reporter constructs containing $\sim 1 \mathrm{~kb}$ of DNA spanning the 478B14-848 and 420M9-1395 HapICE repeats, human SRY was cotransfected at $300 \mathrm{ng}(+\operatorname{SRY}(1: 1))$ and $900 \mathrm{ng}(+\mathrm{SRY}(1: 3))$. The wild-type and schizophrenia-associated novel 5-SNP haplotypes are represented by the light and dark gray bars, respectively. The normalized average values from two triplicate experiments are given.

the wild-type allele (Supplementary Figure 5), showing a direct effect of at least one of the novel DNA variants on transcription factor-binding efficiency. This suggests that the novel SNP haplotype may accentuate the ability of SRY to downregulate transcriptional activity at this enhancer, at least in this context.

\section{Discussion}

NRG1, first identified as a schizophrenia susceptibility gene by linkage studies, has a relatively common risk haplotype in the $5^{\prime}$ promoter region; however, the mechanism through which this specific risk haplotype may increase susceptibility to mental illness is controversial. Our study has shown that the $5^{\prime}$ upstream regions of $N R G 1$ have a relatively high nucleotide diversity as compared with the $5^{\prime}$ regions of many other human genes previously investigated, ${ }^{32-34}$ placing NRG1 in the top $15 \%$ of those investigated. This suggests that the regulatory regions of NRG1 may be relatively unstable and a hotspot for genetic change. Furthermore, people with schizophrenia demonstrate a higher novel variant load than controls, 
particularly in the HapICE LD block; however, the high variant load was not linked to the specific risk haplotype. Observations of a higher mutational load may fit with other mechanistic studies that suggest the genomes of people who develop schizophrenia are more susceptible to DNA damage or mistakes in DNA repair. ${ }^{35}$ Indeed a recent study, suggests that rare de novo mutations were more common in schizophrenia genomes. ${ }^{36}$ However, as peripheral tissue samples were not collected alongside the brain tissue used in this study, we are unable to determine whether the high novel variant load we observed in NRG1 in schizophrenia is brain specific. The presence of the novel 5-SNP haplotype in DNA from peripheral blood in an independent cohort implies a germline rather than somatic origin for this particular haplotype, nevertheless future studies sampling additional tissue sources from individual patients would be beneficial in determining the mechanism of the increased nucleotide diversity in NRG1.

Importantly, this study provides the first demonstrated link between the HapICE risk haplotype and increased NRG1 type III mRNA expression levels in the human frontal cortex. A previous study linking rs7014762 genotype with type III expression reported that the minor allele (designated $\mathrm{T}$ ) was associated with schizophrenia, and that the minor allele resulted in a reduced expression of type III mRNA. ${ }^{15}$ In support of this finding, Nicodemus et al. ${ }^{15}$ incorrectly stated that the same minor allele was also overtransmitted in bipolar disorder in the study by Georgieva et al. ${ }^{37}$; whereas it appears that it was the major allele that was overtransmitted. We show the same direction of effect of the minor allele (designated A) on type III expression, with reduced expression of minor allele homozygotes compared with major allele homozygotes. However, our data and that of others, ${ }^{37}$ shows that it is the major allele of rs7014762 that lies on the schizophreniaassociated HapICE risk haplotype and the major allele that is significantly overtransmitted to bipolar patients. ${ }^{37}$ Hence, we propose a novel mechanism of risk for psychosis, whereby an increase, rather than a decrease, in type III mRNA is linked to NRG1 genetic risk. Consistent with this observation is that increased type III mRNA levels correlate with an earlier age of onset and hence a more severe phenotype. Interestingly, a study by Papiol et al. ${ }^{38}$ indicates an age of onset effect of the 'protective' alleles at SNP8NRG221533 and SNP8NRG243177, where homozygous individuals (TT and $\mathrm{CC}$, respectively) show a later age of onset. This is conceptually consistent with our finding that the HapICE risk alleles lead to an earlier age of onset, by increasing type III expression.

Our finding raises the need to more fully understand the role of NRG1 type III, the most brain-abundant and functionally unique form of NRG1. NRG1 type III acts in a contactdependant manner, is expressed higher in deeper cortical layers and can be found on axons of projection neurons. ${ }^{39,40}$ Furthermore, NRG1 type III is membrane bound and thought to be uniquely capable of back signaling to the nucleus by proteolytic processing and release of a transcriptionally active intracytoplasmic domain that increases neuronal survival. ${ }^{41}$ We previously found evidence of increased NRG1-intracytoplasmic domain domain in the DLPFC of people with schizophrenia. ${ }^{42}$ Recently, an amino-acid sub- stitution within the intramembranous domain of NRG1 was associated with schizophrenia ${ }^{43}$ and this change abolishes intracytoplasmic domain cleavage and leads to decreased branching and growth of cortical neuronal dendrites. ${ }^{3}$ Commonly employed NRG1 type III mouse models use a heterozygous transmembrane domain knockout $^{8}$ or a cystine-rich domain knockout, ${ }^{44}$ both of which model a reduction in type III NRG1 expression while maintaining expression of the soluble isoforms. Our data, which links type III overexpression with disease risk, suggests that a NRG1 type III hypermorphic mouse ${ }^{45}$ may help to elucidate the molecular mechanism of NRG1-related disease risk. Further work is also needed to clarify how increased type III mRNA may further be linked to changes in protein levels, NRG1-intracytoplasmic domain formation and cortical function.

Another unique finding of our study was the discovery of a novel 5-SNP haplotype in intron 1 that was present in $10 \%$ of cases with schizophrenia (4/37 individuals vs $0 / 37$ controls). This haplotype was detected in peripheral blood from one Australian control from a wider population sample, indicating that it is both rare, and may not be exclusive to the brain or disease. However, without longitudinal follow-up of this subject, we cannot rule out that this haplotype is unique to schizophrenia, as some individuals can be diagnosed later in life. ${ }^{46}$ As this novel SNP haplotype was found in DNA derived from both blood and brain, it is likely that these changes do not reflect somatic changes; however, further studies comparing genetic diversity across different tissue types from the same individual are necessary to more directly address the issue of how ubiquitous unique and rare variants in the NRG1 gene promoter may be.

If this novel 5-SNP haplotype is causal, then it should show direct functional effects. In support of this, the 420M9-1395 region-containing $3 / 5$ novel SNPs-was a potent driver of gene expression in vitro. Furthermore, we found that SRY, a sox-like male specific transcription factor, was able to downregulate activity of the 420M9-1395 enhancer and that repression was more extreme with the novel schizophreniaassociated haplotype. Although we were not able to directly link the novel 5-SNP haplotype to NRG1 isoform expression in the brain because of the rarity of the haplotype, we did find a more common haplotype that spanned the 420M9-1395 enhancer region that significantly decreased NRG1 type II expression. Further examination of the transcriptional effects of this enhancer may elucidate whether it is important in driving dysregulation of NRG1 transcripts in certain individuals, particularly the increased type I and decreased type II ratio, which has been reported previously. ${ }^{11}$ In this study, we did not find large changes in any NRG1 isoform with diagnosis alone, however, it may be that heterogeneity in cause and course of schizophrenia or laterality effects may contribute to the difficulties in reproducing mRNA expression changes across independent cohorts.

Postmortem studies such as this do not aim to establish genetic association with increased risk for schizophrenia, but rather aim to explore the link between risk haplotype and changes in gene expression and mutational diversity. Thus an intrinsic limitation of our study is the power of the cohort for detecting significant genetic effects, and hence the findings presented herein should be considered preliminary and 
require replication. This is illustrated by the lack of significant association of the 5-SNP HapICE risk haplotype in our cohort, despite a similar frequency difference between cases and controls in the Australian postmortem cohort and the Icelandic population $^{8}$ (5.4\% vs $7.0 \%$, respectively). In addition, the high nucleotide diversity observed here should be interpreted with caution, as we have not surveyed the entire gene, and have observed a relatively small number of chromosomes. The significance of this increased nucleotide diversity will become apparent with forthcoming next-generation sequencing studies in larger population-based cohorts. Although our postmortem sample is limited to detect statistically significant differences at the genetic level, it is one of the larger ethnically matched Caucasian postmortem brain studies published to date. The earlier studies of Nicodemus et al. ${ }^{15}$ and Law et al. ${ }^{13}$ used a cohort of mixed ethnicity: 44 schizophrenia cases (55\% African American; 45\% Caucasian) and 88 controls (60\% African American; 28\% Caucasian). The Hashimoto et al. ${ }^{11}$ cohort was largely African American with 19 schizophrenia cases (74\% African American; 26\% Caucasian) and 20 controls (85\% African American, 15\% Caucasian). The Parlapani et al. study ${ }^{23}$ used a European Caucasian cohort that included 8 controls and 11 cases for BA10. Given the high genetic diversity in the African American genome as compared with European Caucasians, it is possible that genetic effects observed in the African American population will not be replicated in Caucasians and vice versa.

Although there is still ongoing debate as to the robustness of the genetic association implicating NRG1 as a schizophrenia susceptibility gene, functionally NRG1/ErbB3 is critical for multiple stages of Schwann cell development, ${ }^{47-49}$ and its role in promoting the development of myelin forming cells is now recognized to include oligodendrocytes. Not only are NRG1 and various ErbB receptors expressed in oligogenic zones, ${ }^{2,50}$ but NRG1 can induce the division ${ }^{51}$ and/or promote the differentiation of oligodendrocyte and neuronal precursors in vitro. ${ }^{52-55}$ In particular, cell specific overexpression of NRG1 type III leads to increased myelination in mice, ${ }^{45,56}$ although how this relates to findings in schizophrenia, which shows deficits in molecular markers of myelin or disrupted integrity of white matter, is unclear.

Interestingly, the NRG1-ErbB4 pathway appears critical to the proper development and migration of cortical inhibitory interneurons. ${ }^{2,4,5}$ Soluble NRG1 (types I and II) act as cortical chemoattractants, whereas the membrane bound form (type III) acts in a contact dependant way to help guide migration along certain paths. ${ }^{5}$ Once developing interneurons reach the cortex, axons expressing NRG1 type III can contact postsynaptic ErbB4 and regulate the formation of excitatory synapses from pyramidal neurons onto inhibitory neuron dendrites. ${ }^{4}$ Cortical interneurons are consistently found to be deficient in the prefrontal cortex of people with schizophrenia $^{57}$ and are also abnormal in our cohort. ${ }^{58,59}$ Furthermore, NRG1-induced migration was found to be altered in lymphoblasts from patients with schizophrenia. ${ }^{60}$ More studies are needed to link the pathology of interneuron migration, differentiation and survival to abnormalities in NRG1 in the brains of patients with schizophrenia.

Our findings offer further evidence that abnormal expression of NRG1 isoforms in DLPFC may be related to the pathophysiology of schizophrenia. We suggest a novel mechanism of risk, involving increased NRG1 type III expression in schizophrenia. Furthermore, we suggest that the mechanism by which sequence variation within NRG1 imparts risk to schizophrenia is not solely driven by one SNP, but may relate more to diversity in the genetic neighborhood and accumulation of nucleotide changes. This is fueled by our observation that sequence variation within NRG1 is high and varies even more in the disease state. The implications of our results at the phenotypic level are unknown, but they are at least conceptually consistent with evidence that schizophrenia involves genetic abnormalities in developmental/plasticityrelated processes involving NRG1.

\section{Conflict of interest}

The authors declare no conflict of interest.

Acknowledgements. We thank our Neuroscience Research Australia colleagues, Dr Sinthuja Sivagnanasundaram for GPCR assistance and quantification of EGF $\alpha$ and $\beta$ isoforms; Shan-Yuan Tsai, Duncan Sinclair and Anna Heath for qPCR assistance, particularly housekeeper normalization; Dr Jenny Wong and Heng Woon for screening and cloning the wild-type and mutant 848 and 1395 PCR products into luciferase reporter constructs. Professor Andrew Sinclair and Dr Thomas Ohnesorg (Murdoch Children's Research Institute, Royal Children's Hospital, Parkville, Victoria, Australia) supplied the human SRY construct used in cotransfection studies for luciferase assays. The recruitment of Australian controls was part of the Study on Low-Prevalence (psychotic) Disorders from the National Survey of Mental Health and Wellbeing, Australia, 1997-1998. This work was supported by Grants from the National Health and Medical Research Council of Australia (630452 to CSW and JMF; 630574 to JMF, CSW and PRS) and the Australian Schizophrenia Research Institute. CSW was supported by the Schizophrenia Research Institute (utilizing funding from NSW Health and the Macquarie Group Foundation), Neuroscience Research Australia and the University of New South Wales.

1. Mei L, Xiong WC. Neuregulin 1 in neural development, synaptic plasticity and schizophrenia. Nat Rev Neurosci 2008; 9: 437-452.

2. Anton ES, Ghashghaei HT, Weber JL, McCann C, Fischer TM, Cheung ID et al. Receptor tyrosine kinase ErbB4 modulates neuroblast migration and placement in the adult forebrain. Nat Neurosci 2004; 7 : 1319-1328.

3. Chen Y, Hancock ML, Role LW, Talmage DA. Intramembranous valine linked to schizophrenia is required for neuregulin 1 regulation of the morphological development of cortical neurons. J Neurosci 2010; 30: 9199-9208.

4. Fazzari P, Paternain AV, Valiente M, Pla R, Lujan R, Lloyd K et al. Control of cortical GABA circuitry development by Nrg1 and ErbB4 signalling. Nature 2010; 464: 1376-1380.

5. Flames N, Long JE, Garratt AN, Fischer TM, Gassmann M, Birchmeier C et al. Short- and long-range attraction of cortical GABAergic interneurons by neuregulin-1. Neuron 2004; 44: 251-261.

6. Harrison PJ, Law AJ. Neuregulin 1 and schizophrenia: genetics, gene expression, and neurobiology. Biol Psychiatry 2006; 60: 132-140.

7. Munafo MR, Thiselton DL, Clark TG, Flint J. Association of the NRG1 gene and schizophrenia: a meta-analysis. Mol Psychiatry 2006; 11: 539-546.

8. Stefansson H, Sigurdsson E, Steinthorsdottir V, Bjornsdottir S, Sigmundsson T, Ghosh S et al. Neuregulin 1 and susceptibility to schizophrenia. Am J Hum Genet 2002; 71: 877892.

9. Walker RM, Christoforou A, Thomson PA, McGhee KA, Maclean A, Muhleisen TW et al. Association analysis of Neuregulin 1 candidate regions in schizophrenia and bipolar disorder. Neurosci Lett 2010; 478: 9-13.

10. Munafo MR, Attwood AS, Flint J. Neuregulin 1 genotype and schizophrenia. Schizophr Bull 2008; 34: 9-12.

11. Hashimoto R, Straub RE, Weickert CS, Hyde TM, Kleinman JE, Weinberger DR. Expression analysis of neuregulin-1 in the dorsolateral prefrontal cortex in schizophrenia. Mol Psychiatry 2004; 9: 299-307.

12. Krug A, Markov V, Krach S, Jansen A, Zerres K, Eggermann $T$ et al. The effect of Neuregulin 1 on neural correlates of episodic memory encoding and retrieval. Neuroimage 2010; 53: 985-991. 
13. Law AJ, Lipska BK, Weickert CS, Hyde TM, Straub RE, Hashimoto R et al. Neuregulin 1 transcripts are differentially expressed in schizophrenia and regulated by $5^{\prime}$ SNPs associated with the disease. Proc Natl Acad Sci USA 2006; 103: 6747-6752.

14. Mata I, Perez-Iglesias R, Roiz-Santianez R, Tordesillas-Gutierrez D, Gonzalez-Mandly A, Vazquez-Barquero $\mathrm{JL}$ et al. A neuregulin 1 variant is associated with increased lateral ventricle volume in patients with first-episode schizophrenia. Biol Psychiatry 2009; 65: 535-540.

15. Nicodemus KK, Law AJ, Luna A, Vakkalanka R, Straub RE, Kleinman JE et al. A 5 promoter region SNP in NRG1 is associated with schizophrenia risk and type III isoform expression. Mol Psychiatry 2009; 14: 741-743.

16. Schmechtig A, Vassos E, Kumari V, Hutton SB, Collier DA, Morris RG et al. Association of Neuregulin 1 rs3924999 genotype with antisaccades and smooth pursuit eye movements. Genes Brain Behav 2010; 9: 621-627.

17. Smyrnis N, Kattoulas E, Stefanis NC, Avramopoulos D, Stefanis CN, Evdokimidis I. Schizophrenia-Related Neuregulin-1 Single-Nucleotide Polymorphisms Lead to Deficient Smooth Eye Pursuit in a Large Sample of Young Men. Schizophr Bull 2011; 37: 822-831.

18. Law AJ, Kleinman JE, Weinberger DR, Weickert CS. Disease-associated intronic variants in the ErbB4 gene are related to altered ErbB4 splice-variant expression in the brain in schizophrenia. Hum Mol Genet 2007; 16: 129-141.

19. Nicodemus KK, Luna A, Vakkalanka R, Goldberg T, Egan M, Straub RE et al. Further evidence for association between ErbB4 and schizophrenia and influence on cognitive intermediate phenotypes in healthy controls. Mol Psychiatry 2006; 11: 1062-1065.

20. Norton N, Moskvina V, Morris DW, Bray NJ, Zammit S, Williams NM et al. Evidence that interaction between neuregulin 1 and its receptor erbB4 increases susceptibility to schizophrenia. Am J Med Genet B Neuropsychiatr Genet 2006; 141B: 96-101.

21. Silberberg G, Darvasi A, Pinkas-Kramarski R, Navon R. The involvement of ErbB4 with schizophrenia: association and expression studies. Am J Med Genet B Neuropsychiatr Genet 2006; 141B: 142-148.

22. Walsh T, McClellan JM, McCarthy SE, Addington AM, Pierce SB, Cooper GM et al. Rare structural variants disrupt multiple genes in neurodevelopmental pathways in schizophrenia. Science 2008; 320: 539-543.

23. Parlapani E, Schmitt A, Wirths O, Bauer M, Sommer C, Rueb U et al. Gene expression of neuregulin-1 isoforms in different brain regions of elderly schizophrenia patients. World $J$ Biol Psychiatry 2010; 11(2 Part 2): 243-250.

24. Hahn CG, Wang HY, Cho DS, Talbot K, Gur RE, Berrettini WH et al. Altered neuregulin 1erbB4 signaling contributes to NMDA receptor hypofunction in schizophrenia. Nat Med 2006; 12: 824-828.

25. Boer S, Berk M, Dean B. Levels of neuregulin 1 and 3 proteins in Brodmann's area 46 from subjects with schizophrenia and bipolar disorder. Neurosci Lett 2009; 466: 27-29.

26. Shibuya M, Komi E, Wang R, Kato T, Watanabe Y, Sakai M et al. Measurement and comparison of serum neuregulin 1 immunoreactivity in control subjects and patients with schizophrenia: an influence of its genetic polymorphism. J Neural Transm 2010; 117: 887-895.

27. Stefansson H, Sarginson J, Kong A, Yates P, Steinthorsdottir V, Gudfinnsson E et al. Association of neuregulin 1 with schizophrenia confirmed in a Scottish population. Am J Hum Genet 2003; 72: 83-87.

28. Weickert CS, Miranda-Angulo AL, Wong J, Perlman WR, Ward SE, Radhakrishna V et al. Variants in the estrogen receptor alpha gene and its mRNA contribute to risk for schizophrenia. Hum Mol Genet 2008; 17: 2293-2309.

29. Weickert CS, Sheedy D, Rothmond DA, Dedova I, Fung S, Garrick T et al. Selection of reference gene expression in a schizophrenia brain cohort. Aust N Z J Psychiatry 2010; 44: $59-70$.

30. Jablensky A, McGrath J, Herrman H, Castle D, Gureje O, Evans M et al. Psychotic disorders in urban areas: an overview of the Study on Low Prevalence Disorders. Aust NZ J Psychiatry 2000; 34: 221-236.

31. Fullerton JM, Willis-Owen SA, Yalcin B, Shifman S, Copley RR, Miller SR et al. Humanmouse quantitative trait locus concordance and the dissection of a human neuroticism locus. Biol Psychiatry 2008; 63: 874-883.

32. Licinio J, Dong C, Wong ML. Novel sequence variations in the brain-derived neurotrophic factor gene and association with major depression and antidepressant treatment response. Arch Gen Psychiatry 2009; 66: 488-497.

33. Halushka MK, Fan JB, Bentley K, Hsie L, Shen N, Weder A et al. Patterns of singlenucleotide polymorphisms in candidate genes for blood-pressure homeostasis. Nat Genet 1999; 22: 239-247.

34. Schneider JA, Pungliya MS, Choi JY, Jiang R, Sun XJ, Salisbury BA et al. DNA variability of human genes. Mech Ageing Dev 2003; 124: 17-25.

35. Catts VS, Catts SV, Jablensky A, Chandler D, Shannon Weickert C, Lavin MF. Evidence of aberrant DNA damage response signalling but normal rates of DNA repair in dividing lymphoblasts from patients with schizophrenia. World J Biol Psychiatry 2012; 13: 114-125.

36. Xu B, Roos JL, Dexheimer P, Boone B, Plummer B, Levy $\mathrm{S}$ et al. Exome sequencing supports a de novo mutational paradigm for schizophrenia. Nat Genet 2011; 43: 864-868.

37. Georgieva L, Dimitrova A, Ivanov D, Nikolov I, Williams NM, Grozeva D et al. Support for neuregulin 1 as a susceptibility gene for bipolar disorder and schizophrenia. Biol Psychiatry 2008; 64: 419-427.

38. Papiol S, Begemann M, Rosenberger A, Friedrichs H, Ribbe K, Grube S et al. A phenotypebased genetic association study reveals the contribution of neuregulin1 gene variants to age of onset and positive symptom severity in schizophrenia. Am J Med Genet B Neuropsychiatr Genet 2011; 156B: 340-345

39. Hancock ML, Canetta SE, Role LW, Talmage DA. Presynaptic type III neuregulin1-ErbB signaling targets alpha7 nicotinic acetylcholine receptors to axons. J Gen Physiol 2008; 131: i4.

40. Shamir A, Buonanno A. Molecular and cellular characterization of neuregulin-1 type IV isoforms. J Neurochem 2010; 113: 1163-1176.

41. Bao J, Wolpowitz D, Role LW, Talmage DA. Back signaling by the Nrg-1 intracellular domain. J Cell Biol 2003; 161: 1133-1141.

42. Chong VZ, Thompson M, Beltaifa S, Webster MJ, Law AJ, Weickert CS. Elevated neuregulin-1 and ErbB4 protein in the prefrontal cortex of schizophrenic patients. Schizophr Res 2008; 100: 270-280.

43. Walss-Bass C, Liu W, Lew DF, Villegas R, Montero P, Dassori A et al. A novel missense mutation in the transmembrane domain of neuregulin 1 is associated with schizophrenia. Biol Psychiatry 2006; 60: 548-553.

44. Wolpowitz D, Mason TB, Dietrich P, Mendelsohn M, Talmage DA, Role LW. Cysteine-rich domain isoforms of the neuregulin-1 gene are required for maintenance of peripheral synapses. Neuron 2000; 25: 79-91.

45. Michailov GV, Sereda MW, Brinkmann BG, Fischer TM, Haug B, Birchmeier C et al. Axonal neuregulin-1 regulates myelin sheath thickness. Science 2004; 304: 700-703.

46. Wynn Owen PA, Castle DJ. Late-onset schizophrenia: epidemiology, diagnosis, management and outcomes. Drugs Aging 1999; 15: 81-89.

47. Meyer D, Birchmeier C. Multiple essential functions of neuregulin in development. Nature 1995; 378: 386-390.

48. Riethmacher D, Sonnenberg-Riethmacher E, Brinkmann V, Yamaai T, Lewin GR, Birchmeier $\mathrm{C}$. Severe neuropathies in mice with targeted mutations in the ErbB3 receptor. Nature 1997; 389: 725-730.

49. Syed N, Reddy K, Yang DP, Taveggia C, Salzer JL, Maurel P et al. Soluble neuregulin-1 has bifunctional, concentration-dependent effects on Schwann cell myelination. $J$ Neurosci 2010; 30: 6122-6131.

50. Chong VZ, Webster MJ, Rothmond DA, Weickert CS. Specific developmental reductions in subventricular zone ErbB1 and ErbB4 mRNA in the human brain. Int J Dev Neurosci 2008; 26: 791-803.

51. Canoll PD, Musacchio JM, Hardy R, Reynolds R, Marchionni MA, Salzer JL. GGF/ neuregulin is a neuronal signal that promotes the proliferation and survival and inhibits the differentiation of oligodendrocyte progenitors. Neuron 1996; 17: 229-243.

52. Vartanian T, Corfas G, Li Y, Fischbach GD, Stefansson K. A role for the acetylcholine receptor-inducing protein ARIA in oligodendrocyte development. Proc Natl Acad Sci USA 1994; 91: 11626-11630.

53. Raabe TD, Clive DR, Wen D, DeVries GH. Neonatal oligodendrocytes contain and secrete neuregulins in vitro. J Neurochem 1997; 69: 1859-1863.

54. Raabe TD, Francis A, DeVries GH. Neuregulins in glial cells. Neurochem Res 1998; 23: 311-318.

55. Calaora V, Rogister B, Bismuth K, Murray K, Brandt H, Leprince $\mathrm{P}$ et al. Neuregulin signaling regulates neural precursor growth and the generation of oligodendrocytes in vitro. J Neurosci 2001; 21: 4740-4751.

56. Brinkmann BG, Agarwal A, Sereda MW, Garratt AN, Muller T, Wende H et al. Neuregulin-1/ ErbB signaling serves distinct functions in myelination of the peripheral and central nervous system. Neuron 2008; 59: 581-595.

57. Lewis DA, Hashimoto T, Volk DW. Cortical inhibitory neurons and schizophrenia. Nat Rev Neurosci 2005; 6: 312-324.

58. Fung SJ, Webster MJ, Sivagnanasundaram S, Duncan C, Elashoff M, Weickert CS. Expression of interneuron markers in the dorsolateral prefrontal cortex of the developing human and in schizophrenia. Am J Psychiatry 2010; 167: 1479-1488.

59. Yang Y, Fung SJ, Rothwell A, Tianmei S, Weickert CS. Increased interstitial white matter neuron density in the dorsolateral prefrontal cortex of people with schizophrenia. Biol Psychiatry 2011; 69: 63-70.

60. Sei Y, Ren-Patterson R, Li Z, Tunbridge EM, Egan MF, Kolachana BS et al. Neuregulin1induced cell migration is impaired in schizophrenia: association with neuregulin1 and catechol-o-methyltransferase gene polymorphisms. Mol Psychiatry 2007; 12: 946-957.
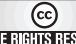
licensed under the Creative Commons Attribution-Noncommercial-No Derivative Works 3.0 Unported License. To view a copy of this license, visit http://creativecommons.org/licenses/by-nc-nd/3.0/

\section{Supplementary Information accompanies the paper on the Translational Psychiatry website (http://www.nature.com/tp)}

